\title{
Discretization of electronic states in large InAsP/InP multilevel quantum dots probed by scanning tunneling spectroscopy
}

\author{
B. Fain, ${ }^{1}$ I. Robert-Philip, ${ }^{1}$ A. Beveratos,${ }^{1}$ C. David, ${ }^{1}$ Z.Z. Wang, ${ }^{1}$ I. Sagnes,${ }^{1}$ and J.C. Girard ${ }^{1}$ \\ ${ }^{1}$ Laboratoire de Photonique et de Nanostructures LPN-CNRS UPR20, Route de Nozay, 91460 Marcoussis, France \\ ${ }^{*}$ Corresponding author: jean-christophe.girard@lpn.cnrs.fr
}

\begin{abstract}
The topography and the electronic structure of InAsP/InP quantum dots are probed by crosssectionnal scanning tunneling microscopy and spectroscopy. The study of the local density of states in such large quantum dots confirms the discrete nature of the electronic levels whose wavefunctions are measured by differential conductivity mapping. Due to their large dimensions, the energy separation between the discrete electronic levels is low, allowing for quantization both in the lateral and growth directions as well as the observation of the harmonicity of the dot lateral potential.
\end{abstract}

PACS numbers: 73.22.Dj ; 81.07.Ta ; 68.37.Ef

Semiconductor quantum dots (QDs) offer promising opportunities as sources of non-classical states of light [1-3] or as gain media in microcavity lasers [4]. Whereas most of the reports on QDs use the $\operatorname{In}(\mathrm{Ga}) \mathrm{As} / \mathrm{GaAs}$ system, $\operatorname{InAs}(\mathrm{P}) / \mathrm{InP}$ QDs are of great interest because of their broad emission wavelength tunability, which includes the O-band and C-band telecommunications window around $1.3 \mu \mathrm{m}$ and $1.55 \mu \mathrm{m}$ respectively [5-8]. Yet, the large lateral size of these QDs, more particularly the ones emitting in the C-band, raises the issue whether they can still be considered as 0D quantum systems with discrete electronic energy levels [9]. This issue requires a better knowledge of the local electronic structure of individual dots, in correspondance with their structural properties. This can be obtained by scanning tunneling microscopy and spectroscopy (STM/STS) already applied to semiconductor nanostructures such as quantum wells [10] and InAs/GaAs quantum dots [11-13]. In this letter, we report a cross-sectional STM/STS study of cleaved InAsP/InP(001) QDs at $4 \mathrm{~K}$, revealing up to 9 discrete equidistant electronic levels. These electronic states are identified by spatially and energetically resolved mapping of the wavefunctions, indicating quantization both along the base and along the height of the QD. These measurements, supplemented by numerical simulations, confirm the discrete nature of the confined electronic states of the $\mathrm{QD}$ and also reveal the harmonic nature of the QD lateral potential.

The QD sample was grown by low-pressure MetalOrganic Chemical Vapor Deposition on a (001) p-type InP substrate and contains four InAsP quantum dot layers embedded in a thick highly $p$-doped layer $\left(\mathrm{N}_{A}[\mathrm{Zn}]=10^{18} \mathrm{~cm}^{-3}\right)$ [13]. Energy Dispersive X-Ray (EDX) analysis reveals a slight gradient within the InAsP QD, from $\operatorname{InAs}_{0.75} \mathrm{P}_{0.25}$ at the QD bottom and edges to InAs $\mathrm{As}_{0.85} \mathrm{P}_{0.15}$ at the QD top center. Plane-view TEM studies reveal that most of the QDs of this sample present an elliptic shape. Their mean width and height are $40 \pm 15 \mathrm{~nm}$ and $5 \pm 2 \mathrm{~nm}$ respectively. The dot density is of the order of $10^{10} \mathrm{~cm}^{-2}$ per layer
[14]. The sample was cleaved in situ along the (110) surface in a preparation chamber at a base pressure of $7.10^{-11}$ mbar and loaded subsequently in the STM head precooled at low temperature (4K). Figure 1(a) shows a constant-current topographical image in which the measured contrast depends not only on a topographic elastic effect due to strain relaxation after cleavage, but also on an electronic contrast due to the variation in the density of states between the quantum dot and the surrounding material. The cleaved QD displays a large aspect ratio (30 $\mathrm{nm}$ apparent width at the base along the exposed cleaved surface and $3.8 \mathrm{~nm}$ height) and a trapezoidal cross-section with an angle of $28^{\circ}$. Note that the analysis of such images allows for an accurate measurement of the dot height and apparent lateral width but gives no quantitative information on its depth in the [110] direction, since the cleavage plane location is not known.

Our STS analysis is based on the relation between the differential conductance and the local density of states (DoS) of the sample at the position of the tip vertex. In our STS experiments, the differential conductivity $d I / d V_{\text {sample }}$ is directly obtained by using a lock-in technique with a $5 \mathrm{mV}$ modulation $V_{\text {mod }}^{R M S}$ of $V_{\text {sample }}$ at 923 $\mathrm{Hz}$ and a time constant of $30 \mathrm{~ms}$, as described in [12]. Figure 1 shows conductance spectra obtained at different tip positions on the QD surface and on the $\operatorname{InP}(1 \overline{1} 0)$ bare surface with a sample voltage $V_{\text {sample }}$ varying from -0.6 to $1.7 \mathrm{~V}$. The measurement positions are indicated by crosses on the figure 1(f). Measurements on the surrounding $p$-doped InP matrix (Figure 1(b)) reveal the expected local DoS in conventional bulk materials, with the valence band below $-0.2 \mathrm{~V}$ and the conduction band above $1.4 \mathrm{~V}$. The measured bandgap $(\simeq 1.6 \mathrm{~V})$ is bigger than the expected value of $1.42 \mathrm{~V}$ because of tip-induced band bending (TIBB) effects $[15,16]$. The simulations of band bending through the SEMITIP program [17] yield flat band conditions around $V_{\text {sample }} \simeq 1 \mathrm{~V}$ for InP, indicating that TIBB effects are negligible in this region [18] 


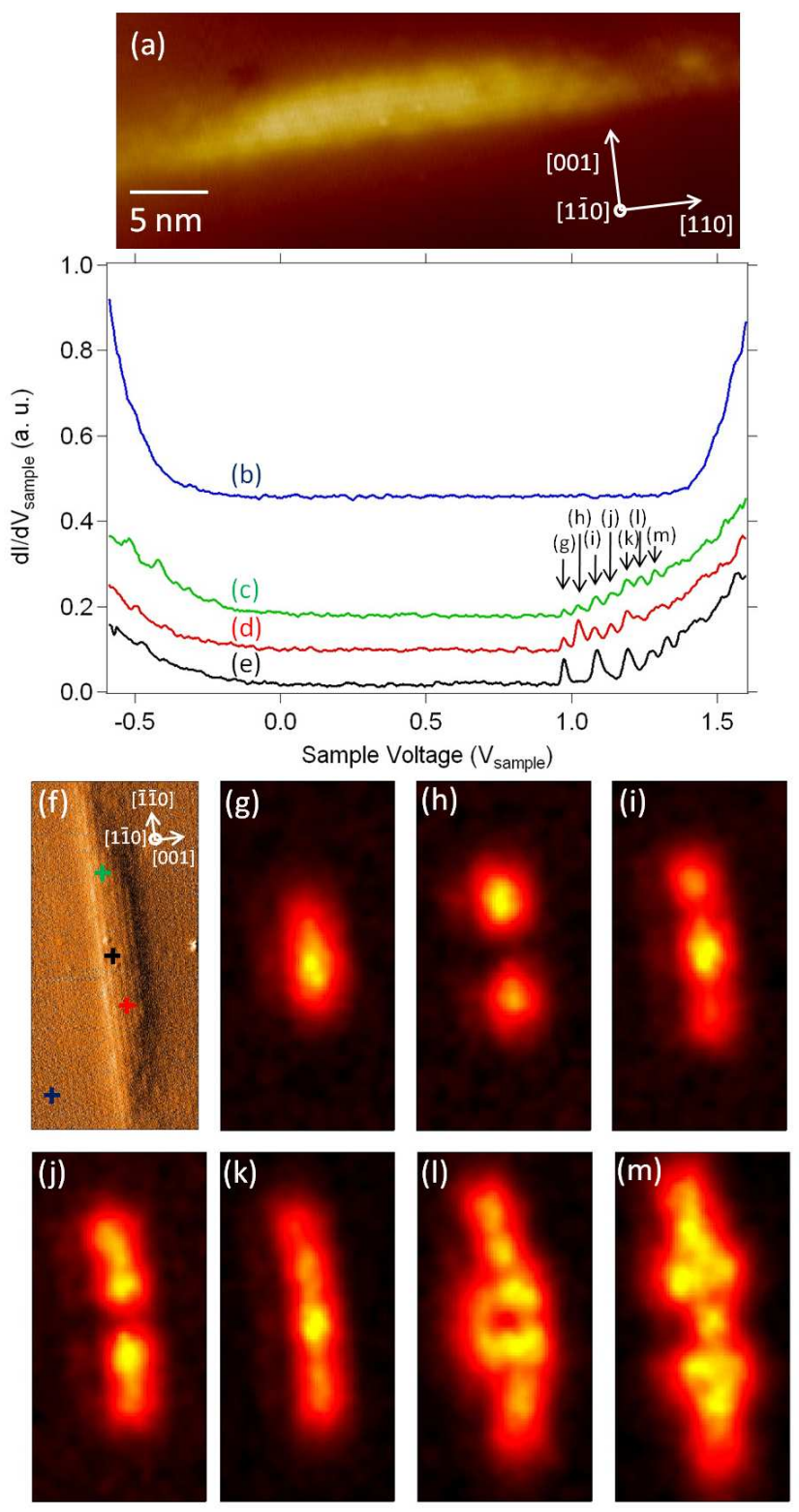

FIG. 1: (Top) (a) X-STM topographic image of an InAsP quantum dot $(V=+1.7 \mathrm{~V} ; I=300 \mathrm{pA})$. The Z-contrast of the dot is $200 \pm 20 \mathrm{pm}$. (b-e) STS measurements obtained at $\mathrm{T}=4 \mathrm{~K}$ on bare $\operatorname{InP}(1 \overline{1} 0)$ surface $(\mathrm{b}$ - dark blue line), and on an InAsP quantum dot at various locations (c - green line, $\mathrm{d}$ - red line, e - black line). The spectra are shifted for clarity. Signal was integrated for $20 \mathrm{~ms}$ at each point.(Bottom (f)) Quantum dot derivative image where the locations of STS spectra (b-e) are indicated by crosses with corresponding colors. (Bottom (g)-(m)) $16 \mathrm{~nm}$ x $32 \mathrm{~nm}$-size $d I / d V_{\text {sample }}$ maps measured respectively at $0.974 \mathrm{~V}, 1.024 \mathrm{~V}, 1.085 \mathrm{~V}, 1.143 \mathrm{~V}$, $1.197 \mathrm{~V}, 1.250 \mathrm{~V}$ and $1.289 \mathrm{~V}$ corresponding to the arrows on the above STS measurements curve. Each picture has its own arbitrary color scale.

whereas they influence strongly the valence band measurement.
Figure 1(c-e) show spectra obtained at different tip positions on the same QD (labeled QD1). These spectra display peaks between 0.9 and $1.3 \mathrm{~V}$. These peaks are related to quantum confinement already studied in QWs $[10]$ for instance. For our QD measurements, each discrete peak corresponds to an electronic state arising from 3D confinement. The full width at half maximum (FWHM) of all these peaks, not limited by the resolution of our setup $\left(\delta E=\sqrt{\left(2.5 e V_{\text {mod }}\right)^{2}+(3.3 k T)^{2}}=13 \mathrm{meV}\right.$ [19]), is about $30 \mathrm{meV}$, of the same order as that observed on colloidal quantum dots [20], self-assembled InAs/GaAs quantum dots [21] or Ag nanopyramid [22]. The broadening of the peaks may arise from electronphonon scattering or surface charge fluctuations [22, 23]. The relative weights of the different peaks depend on the measurement positions. This variation reflects the spatial distribution of the electronic wavefunctions, which may thus be spatially mapped by recording $d I / d V_{\text {sample }}$ spectra on a grid of points on the cleaved QD surface (spatial resolution: $7 \AA$ ). Such maps are presented in Figure 1(g-m) (bottom). They allow for an identification of the dot's successive electronic $(\mathrm{nmp})$ states, where the numbers $n, m$ and $p$ correspond to the number of nodes in the [110], [110] and [001] directions respectively. The first energy levels correspond to $(n 00)$ states: the first peak (g) displays the expected elliptic intensity distribution for the (000) ground state, while the higher energy peaks (h-k) present lobe features with an increasing number of nodes along the [110] direction consistent with $(n 00)$ states. The wave functions bow slightly toward the [001] direction, which may result from the QD shape or the QD gradient. The high-energy maps (l) and (m) display a more complex structure, originating from the superposition of two electronic states: a $(n 00)$ state and a $\left(n^{\prime} 01\right)$ state. This state sequence is supported by the measurement of the integrated density of states obtained on three different QDs (QD1 as well as QD2 and QD3). For the three dots, the measured integrated density of states shown on figure 2 display low energy peaks associated to the $(n 00)$ states, as corroborated by the spatial distribution of the differential conductance. As expected, the energy of the fundamental (000) level decreases with the QD heigth and the energy separation between the successive $(n 00)$ states decreases with the QD size. Moreover, this energy separation is almost constant for each dot, revealing the harmonic nature of the dot potential (see Fig. 2(d)). This integrated DoS also display high energy peaks that lie on a step-like background originating from the superimposed $(n 00)$ and $\left(n^{\prime} 01\right)$ states. The observation of $\left(n^{\prime} 01\right)$ states is due to the relatively large dot heights compared, for instance, with that of InAs/GaAs quantum dots [21]. This results in an energy separation of the order of a few tens meV, in contrast to a few hundreds $\mathrm{meV}$ in the InAs/GaAs material system with similar conduction band offset. The large 


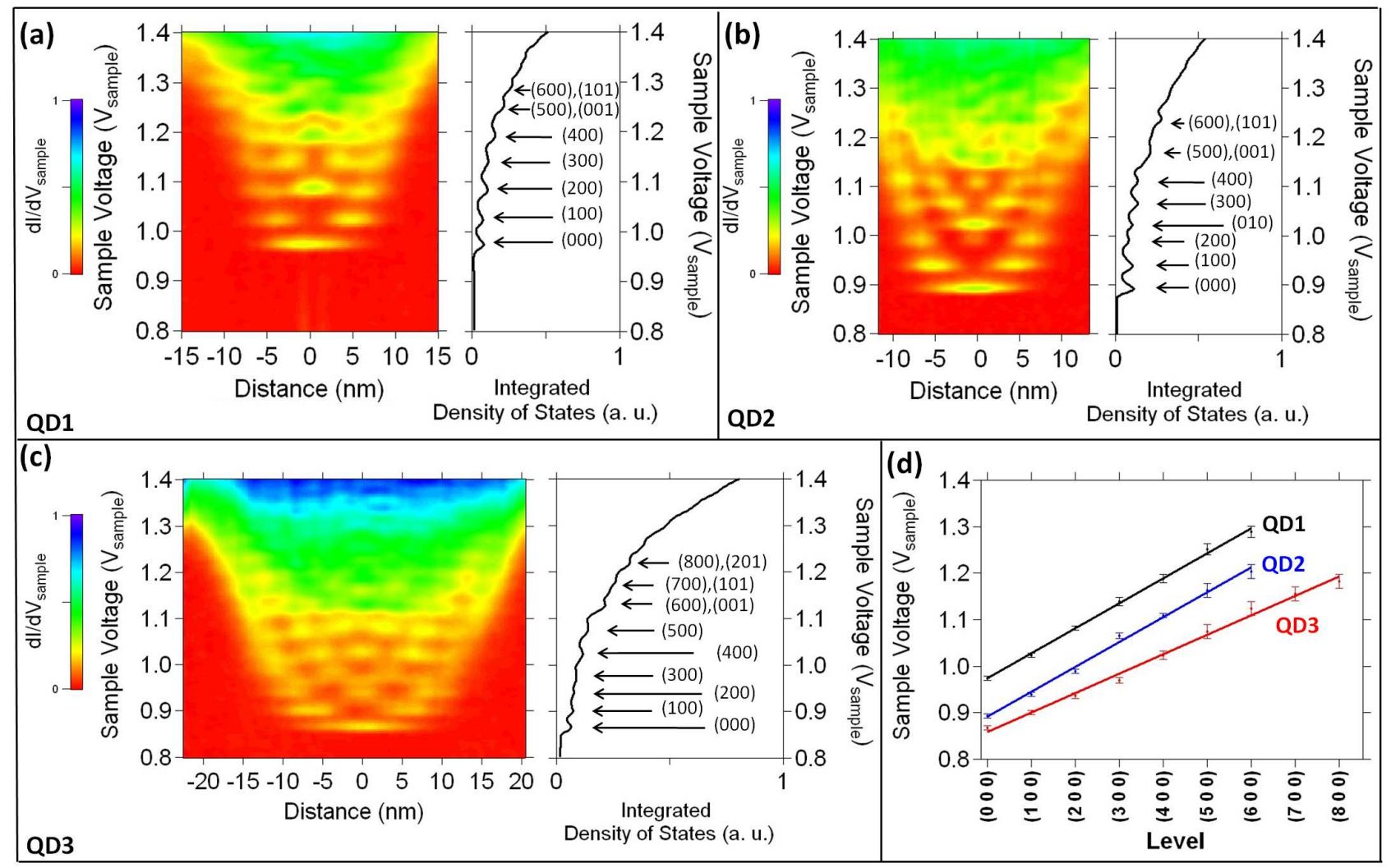

FIG. 2: $d I / d V_{\text {sample }}$ as a function of the applied voltage and of the distance from the dot center along the quantum dot base, measured on three different InAsP quantum dots labeled QD1 (a), QD2 (b) and QD3 (c) with an apparent width and height of $30 \mathrm{~nm}$ and $3.8 \mathrm{~nm}, 30 \mathrm{~nm}$ and $5.9 \mathrm{~nm}, 42 \mathrm{~nm}$ and $5.9 \mathrm{~nm}$ respectively. The observation of the (010) state on QD2 suggests a larger depth for this dot compared with QD1 and QD3. The conductance is averaged along the growth axis over a $10 \mathrm{~nm}$ window centered on the middle of the dot. For each plot, the differential conductance integrated along the dot base is shown (arbitrary scales). The dependence of the $(n 00)$ level energy as a function of the quantum numbers, for the three dots, is shown in $(d)$.

number of electronic states, observed in these dots, has been confirmed also on uncleaved quantum dots through optical spectroscopy [9].

In order to unambiguously identify the state sequence and quantatively support our analysis, finite element simulations of the electronic wavefunctions (Comsol Multiphysics) were carried out.As shown in Fig. 3(a), the QDs are modeled as truncated cylinders. The axis of the cylinder is the [001] growth direction and the truncation plane corresponds to the $(1 \overline{1} 0)$ cleavage plane. The QD is surrounded by InP, except on the side corresponding to the (110) surface which is exposed to vacuum. In order to simulate the trapezoidal cross-section of the $\mathrm{QD}$ and the $\mathrm{QD}$ gradient, the lateral potential of the cylinder was assumed to be progressive with a parabolic dependence on distance [24], as suggested by the equidistant $(n 00)$ levels. Our calculations do not account for possible strain or surface effects [11] and the observed $30 \mathrm{meV}$ broadening of the energy levels is introduced numerically. Last, many-body effects [25] have been assumed to be negligible. This was confirmed by repeating the experiments with a $100 \mathrm{pA}$ tunneling current, which corresponds to an electron injection time of $1 \mathrm{~ns}$ close to the electron lifetime in an uncleaved dot [9]. Simulations carried out on QD3 and their experimental counterparts are presented on Fig. 3(b-j). Below $1.1 \mathrm{eV}$ corresponding to the first $(n 00)$ states (with $n=0$ up to 5 ), the simulated wavefunction maps exhibit the same sequence as our measurements and the corresponding energies are in good agreement with our experimental data. Above $1.1 \mathrm{eV}$, the simulated wavefunction maps reproduce the lobe features of our experiments when taking into account the superposition of two close $(n 00)$ and $\left(n^{\prime} 01\right)$ energy levels overlaping due to level broadening.

In conclusion, STS spectra and wavefunction mapping on InAsP/InP quantum dots confirmed the discrete nature of the electronic confinement in such large QDs. Due 


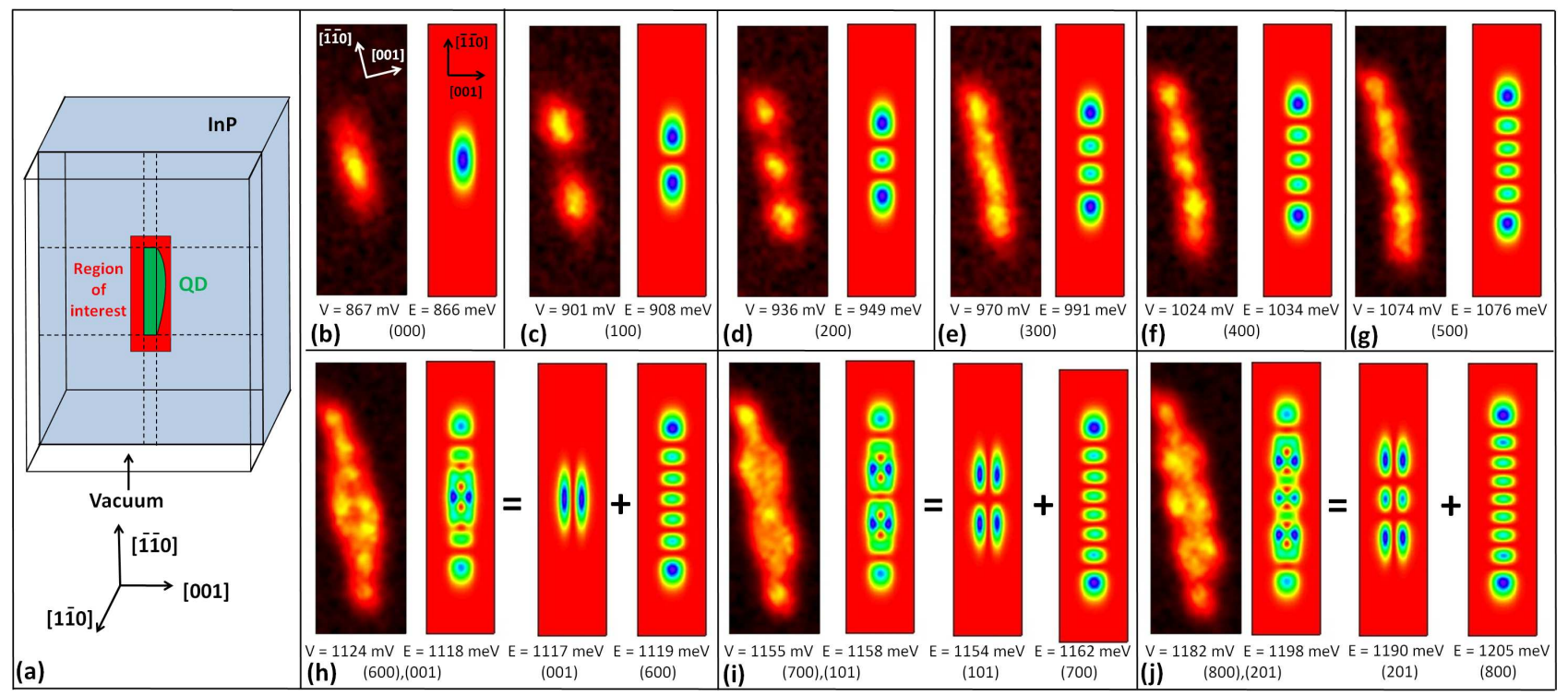

FIG. 3: (a) QD geometry used for simulation. The QD (green) is surrounded by InP (blue) and vacuum. (b-j) Measured $d I / d V_{\text {sample }}$ maps $(15 \mathrm{~nm} \times 45 \mathrm{~nm})$ and simulations of the electronic wavefunction spatial distribution $(15 \mathrm{~nm} \times 60 \mathrm{~nm})$ for the electronic states of QD3. The simulations display the square modulus of the wave function in the plane separating the semiconductor from the vacuum, corresponding to the red area of (a). Calculations assume an apparent width and height of the dot of respectively $56 \mathrm{~nm}$ and $5.6 \mathrm{~nm}$. The dot depth is chosen equal to $5 \mathrm{~nm}$. Each picture has its own arbitrary color scale.

to their large size, these QDs can accomodate a large number of electrons and their electronic levels display a quantization both in the lateral and growth direction. The large number of observed states permits us to model the QD lateral potential as harmonic. Earlier measurements, especially in $\operatorname{In}(\mathrm{Ga}) \mathrm{As} / \mathrm{GaAs}$ QDs, had identified only a few electronic levels $[12,21]$, and could not therefore provide sufficient information to determine the shape of the confining potential. We hope that these results will help in understanding charge effects in InAsP/InP QDs and successfully interpret their macroscopic electronic and optical characteristics, a key element for telecommunications wavelength quantum optics and nanophotonics.

We are grateful to J.-Y. Marzin, I. Abram, G. Rodary, D. Elvira and G. Beaudoin for fruitful discussion. The authors acknowledge financial support from Direction Générale de l'Armement (DGA).

[1] P. Michler, A. Kiraz, C. Becher, W.V. Schoenfeld, P.M. Petroff, L. Zhang, E. Hu and A. Imamoglu, Science 290, 2282 (2000).

[2] C. Santori, M. Pelton, G. Solomon, Y. Dale and Y. Yamamoto, Phys. Rev. Lett. 86, 1502-1505 (2001).

[3] R.M. Stevenson, R.J. Young, P. Atkinson, K. Cooper, D.A. Ritchie and A.J. Shields, Nature 439, 179 (2006).

[4] S. Strauf, K. Hennessy, M.T. Rakher, Y.-S. Choi, A. Badolato, L.C. Andreani, E.L. Hu, P.M. Petroff, and D.
Bouwmeester, Phys. Rev. Lett. 96, 127404 (2006).

[5] D. Elvira, A. Michon, B. Fain, G. Patriarche, G. Beaudoin, I. Robert-Philip, Y. Vachtomin, A.V. Divochiy, K.V. Smirnov, G.N. Goltsman, I. Sagnes, and A. Beveratos, Appl. Phys. Lett. 97, 131907 (2010)

[6] K. Takemoto, Y. Sakuma, S. Hirose, T. Usuki, N. Yokoyama, T. Miyazawa, M. Takatsu and Y. Arakawa, Physica E 26, 185 (2005).

[7] T. Kuroda, Y. Sakuma, K. Sakoda, K. Takemoto and T. Usuki, Appl. Phys. Lett. 91, 223113 (2007).

[8] P.J. van Veldhoven, N. Chauvin, A. Fiore and R. Nötzel, Appl. Phys. Lett. 95, 113110 (2009).

[9] D. Elvira, R. Hostein, B. Fain, L. Monniello, A. Michon, G. Beaudoin, R. Braive, I. Robert-Philip, I. Abram, I. Sagnes, and A. Beveratos, Phys. Rev. B 84, 195302 (2011)

[10] K. Suzuki, K. Kanisawa, C. Janer, S. Perraud, K. Takashina, T. Fujisawa, and Y. Hirayama, Phys. Rev. Lett. 98, 136802 (2007)

[11] B. Grandidier, Y.M. Niquet, B. Legrand, J.P. Nys, C. Priester, and D. Stiévenard, J.M. Gérard, and V. Thierry-Mieg, Phys. Rev. Lett. 85, 1068 (2000).

[12] J. C. Girard, A. Lemaître, A. Miard, C. David, and Z. Z. Wang, J. Vac. Sci. Technol. B 27, 891 (2009)

[13] B. Fain, J. C. Girard, D. Elvira, C. David, G. Beaudoin, A. Beveratos, I. Robert-Philip, I. Sagnes, and Z. Z. Wang, Appl. Phys. Lett. 97, 171903 (2010)

[14] A. Michon, G. Saint-Girons, G. Beaudoin, I. Sagnes, L. Largeau, and G. Patriarche, Appl. Phys. Lett. 87, 253114 (2005)

[15] R. M. Feenstra, G. Meyer, F. Moresco, and K. H. Rieder, Phys. Rev. B 66, 165204 (2002)

[16] G. J. de Raad, D. M. Bruls, P. M. Koenraad, and J. H. 
Wolter, Phys. Rev. B 66, 195306 (2002)

[17] Y. Dong, R.M. Feenstra, M.P Semtsiv, and W.T. Masselink, J. Appl. Phys. 103, 073704 (2008).

[18] M. Weimer, J. Kramar, and J. D. Baldeschwieler, Phys. Rev. B 39, 5572 (1989)

[19] M. Morgenstern, J. Wiebe, F. Marczinowski and R. Wiesendanger, Quantum Materials, NanoScience and Technology 9, 217-243 (2010)

[20] U. Banin, Y. Cao, D. Katz, and O. Millo, Nature (London) 400, 542 (1999)

[21] T. Maltezopoulos, A. Bolz, C. Meyer, C. Heyn, W. Hansen, M. Morgenstern, and R. Wiesendanger, Phys.
Rev. Lett. 91, 196804 (2003)

[22] C. Tournier-Colletta, B. Kierren, Y. Fagot-Revurat, and D. Malterre, Phys. Rev. Lett. 104, 016802 (2010).

[23] L. Jdira, K. Overgaag, R. Stiufiuc, B. Grandidier, C. Delerue, S. Speller and D. Vanmaekelbergh, Phys. Rev. B 77, 205308 (2008)

[24] U. Merkt, J. Huser and M. Wagner, Phys. Rev. B 43 $7320(1991)$

[25] G. Maruccio, M. Janson, A. Schramm, C. Meyer, T. Matsui, C. Heyn, W. Hansen, R. Wiesendanger, M. Rontani and E. Molinari, Nanoletters 7, 2701 (2007) 